\section{Nonpolio Enterovirus Activity during the COVID-19 Pandemic, Taiwan, 2020}

\author{
Shu-Chen Kuo, ${ }^{1}$ Hsiao-Hui Tsou, ${ }^{1}$ Hsiao-Yu Wu, \\ Ya-Ting Hsu, Fang-Jing Lee, Shu-Man Shih, \\ Chao A. Hsiung, Wei J. Chen ${ }^{1}$
}

Author affiliations: National Health Research Institutes, Zhunan, Taiwan

DOI: https://doi.org/10.3201/eid2701.203394

In Taiwan, lower nonpolio enterovirus activity during the coronavirus disease pandemic in 2020 compared with 2014-2019 might be attributable to adherence to nonpharmaceutical interventions. The preventable fraction among unexposed persons indicated that $90 \%$ of nonpolio enterovirus activity might have been prevented during 2014-2019 by adopting the same measures enforced in 2020 .

Nitase onpharmaceutical interventions have been shown to be effective in preventing the spread of infectious diseases. The strict compliance with nonpharmaceutical interventions implemented during the coronavirus disease (COVID-19) pandemic has been associated with a decline in influenza activity in many countries, including Taiwan (1-4). Handwashing, disinfecting frequently touched surfaces, and closure of schools might also be effective against nonpolio enteroviruses (NPEV), which commonly cause a spectrum of illnesses in young populations in Asia (5). We observed lower NPEV activity during the 2019-20 season in Taiwan compared with the average of the 5 previous seasons, which might be attributable to strict compliance with nonpharmaceutical interventions. We further estimated the protective effect that could have been achieved if the population strictly adhered to the same nonpharmaceutical interventions during those previous seasons.

We collected nationwide data on weekly outpatient and emergency department (ED) visits during November 2014-June 2020 from the Taiwan National Infectious Disease Statistics System (https://nidss. cdc.gov.tw) (6). Patients $\geq 15$ years of age were excluded because of their milder symptoms and low number of cases. The original data were transferred from the National Health Insurance program of Taiwan, which covers $>99 \%$ of Taiwan residents (Appendix, https://wwwnc.cdc.gov/EID/article/27/1/203394-App1.pdf). The Institutional Review Board of the National Health Research Institutes approved this study (approval no. EC1051207-R4).

${ }^{1}$ These authors contributed equally to this article.
NPEV activity was measured by using the number of visits that yielded diagnoses of hand, foot, and mouth disease (International Classification of Diseases [ICD], 9th Revision, Clinical Modification, code 074.3 or ICD, 10th Revision, Clinical Modification, code B08.4) or herpangina (ICD, 9th Revision, Clinical Modification, code 074.0 or ICD, 10th Revision, code B08.5). The period from week 47 of 1 year and week 23 of the following year was defined as 1 season. We estimated the change in NPEV activity after the first imported COVID-19 case in Taiwan, when nonpharmaceutical interventions were introduced and enforced, by using a differencein-difference model used in a previous influenza study (Appendix) (4). The total number of outpatient and ED visits for NPEV at baseline was adjusted to eliminate the preintervention differences in NPEV activity between groups (2019-20 season vs. 2014-2019 seasons). The total number of outpatient and ED visits for all disease in different weeks and different years was used for normalization because their numbers decreased after the COVID-19 pandemic. We estimated the preventable fraction among the unexposed $\left(P F_{u}\right)$ to measure the reduction of NPEV that would have been possible in each week of the 2014-2019 seasons, had the same nonpharmaceutical interventions been strictly followed, and adjusted $P F_{u}$ to control for potential confounder (Appendix).

The number of NPEV visits during the 2019-20 season was 81,942 , compared with the average of 205,979 during the 2014-2019 seasons (Appendix Table 1). NPEV activity increased after week 16 across the past 6 seasons except 2019-20, when the earlier low level of weekly activity continued (Figure; Appendix Figure 1). The difference-in-difference analysis revealed that after normalization by visits for NPEV at baseline and for all diseases, NPEV activity during weeks 16-23 in the 2019-20 season was significantly lower than during the same calendar weeks of the 2014-2019 seasons (Appendix Table 2). The lower activity during weeks 16-23 in 2019-20 remained significant across all age groups and hospital settings (Appendix Table 3, 4). The weekly $P F_{u}$ increased from $73 \%$ to $90 \%$ (from $17 \%$ to $71 \%$ for adjusted $P F_{u}$ ) during weeks 16-23 (Table; Appendix Table 5). Similar benefits of the nonpharmaceutical interventions were observed across different age groups of patients and hospital settings (Table; Appendix Table 6).

We observed a significant and persistent decrease of NPEV during the 2019-20 season, which might be attributable to strict compliance with the nonpharmaceutical interventions. Up to $90 \%$ ( $71 \%$ adjusted) of NPEV activity might have been prevented during the 2014-2019 seasons by adopting the same nonpharmaceutical interventions enforced in 2020. Many 


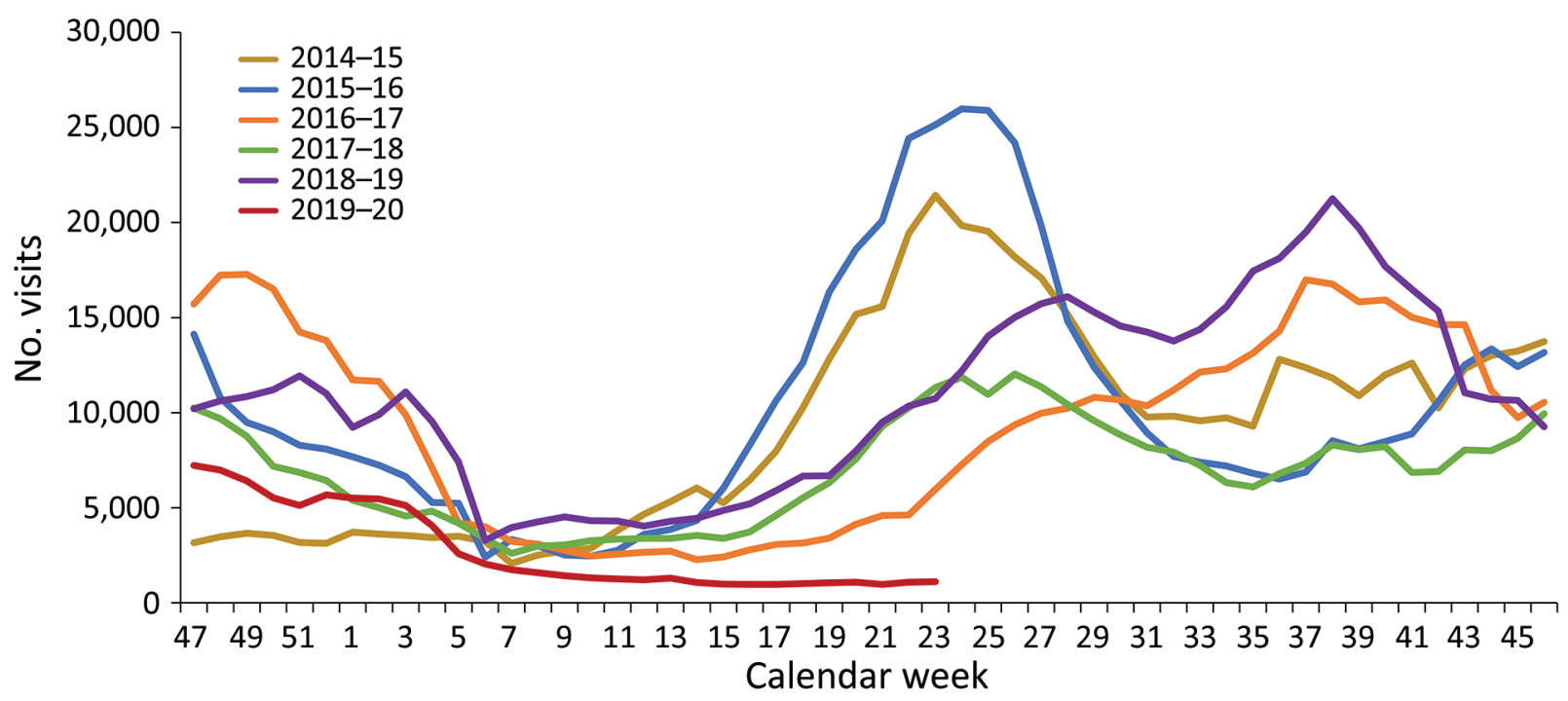

Jan 21: First imported case

Feb 4: Hand-hygiene campaign

Feb 11: Extension of school winter break by 2 weeks

Feb 20: Disinfection of public transportation systems every 8 hours
Apr 1: Guidance for social distancing

Apr 17: Reports of plastic shields used in schools

Mar 17: Reports of campus closed to public

Mar 25: Disinfection of all public places recommended by the Environmental Protection Administration

Feb 25: End of winter break

Temperature monitoring, hand-hygiene education, advocacy of not sharing food or talking at mealtimes, and regular cleaning and disinfecting schools and kindergartens

Figure. Nonpolio enterovirus activity during 2019-20 season compared with the same weeks in the previous 5 seasons in patients $<15$ years of age, Taiwan. The y-axis represents the number of outpatient department and emergency department visits in which a diagnosis of hand, foot and mouth disease or herpangina was made for patients $<15$ years of age.

factors, such as detection bias and healthcare avoidance, might confound our analyses. However, the detection of NPEV is based on symptoms and was less likely to be affected by the COVID-19 pandemic. In addition, COVID-19 had little impact on the surveillance system in Taiwan because $<450$ total COVID-19 cases had been reported as of June 17 and no local cases have been reported since April 12.
Our study is limited by the healthcare avoidance caused by the COVID-19 pandemic (4). The normalization procedure using the number of visits for all diseases in our study and subgroup analyses on ED patients (Appendix Table 4,6) are insufficient to eliminate the impact of healthcare avoidance; active surveillance is required. The effect of individual nonpharmaceutical intervention is difficult to assess. The

\begin{tabular}{|c|c|c|c|c|c|}
\hline \multirow{2}{*}{$\begin{array}{l}\text { Calendar } \\
\text { week }\end{array}$} & \multicolumn{5}{|c|}{ Estimated $P F_{u}(95 \% \mathrm{Cl})$} \\
\hline & Overall & $0-2 y$ & $3-4 y$ & $5-9 y$ & $10-14 \mathrm{y}$ \\
\hline 16 & $0.73(0.67-0.78)$ & $0.55(0.46-0.63)$ & $0.76(0.70-0.81)$ & $0.78(0.73-0.83)$ & $0.74(0.68-0.79)$ \\
\hline 17 & $0.76(0.72-0.80)$ & $0.61(0.54-0.67)$ & $0.79(0.75-0.82)$ & $0.81(0.77-0.85)$ & $0.78(0.73-0.81)$ \\
\hline 18 & $0.79(0.76-0.82)$ & $0.66(0.61-0.70)$ & $0.82(0.79-0.84)$ & $0.84(0.81-0.86)$ & $0.81(0.78-0.83)$ \\
\hline 19 & $0.82(0.80-0.84)$ & $0.70(0.67-0.73)$ & $0.84(0.82-0.86)$ & $0.86(0.84-0.88)$ & $0.83(0.81-0.85)$ \\
\hline 20 & $0.84(0.82-0.86)$ & $0.74(0.71-0.76)$ & $0.86(0.84-0.88)$ & $0.88(0.86-0.89)$ & $0.86(0.84-0.87)$ \\
\hline 21 & $0.86(0.84-0.88)$ & $0.77(0.74-0.80)$ & $0.88(0.86-0.89)$ & $0.89(0.88-0.91)$ & $0.87(0.86-0.89)$ \\
\hline 22 & $0.88(0.86-0.90)$ & $0.80(0.77-0.83)$ & $0.89(0.87-0.91)$ & $0.91(0.89-0.92)$ & $0.89(0.87-0.91)$ \\
\hline 23 & $0.90(0.87-0.91)$ & $0.82(0.79-0.85)$ & $0.91(0.89-0.93)$ & $0.92(0.90-0.94)$ & $0.91(0.88-0.92)$ \\
\hline
\end{tabular}

${ }^{*}$ All values are statistically significant. $P F_{u}$, estimated preventable fraction among the unexposed. 
prolonged winter break might have played a major role in reducing NPEV activity. However, considering the high contagiousness of NPEV, their activity was expected to peak after school reopening if no other interventions were implemented. The persistent low NPEV activity throughout the semester, which began in March 2020, indicated the effectiveness of other interventions.

\section{Acknowledgments}

We thank the Taiwan CDC for making their data publicly available. We also thank Kung-Yee Liang, Huey-Kang Sytwu, Shiow-Ing $\mathrm{Wu}$, and $\mathrm{Yu}$-Chieh Cheng for their valuable comments and support.

This project was supported by intramural grants from the National Health Research Institutes (grant nos. IV-109-PP-01, PH-109-PP-02, and PH-109-GP-02).

\section{About the Author}

Dr. Kuo is an attending physician and associate investigator at the National Institute of Infectious Diseases and Vaccinology, National Health Research Institutes, Taiwan. His primary research interest involves infectious diseases and clinical microbiology.

\section{References}

1. Kuo SC, Shih SM, Chien LH, Hsiung CA. Collateral benefit of COVID-19 control measures on influenza activity, Taiwan. Emerg Infect Dis. 2020;26:1928-30. https:/ / doi.org/10.3201/ eid2608.201192

2. Galvin CJ, Li YJ, Malwade S, Syed-Abdul S. COVID-19 preventive measures showing an unintended decline in infectious diseases in Taiwan. Int J Infect Dis. 2020;98:18-20. https://doi.org/10.1016/j.ijid.2020.06.062

3. Choe YJ, Lee JK. The impact of social distancing on the transmission of influenza virus, South Korea, 2020. Osong Public Health Res Perspect. 2020;11:91-2. https:// doi.org/10.24171/j.phrp.2020.11.3.07

4. Sakamoto H, Ishikane M, Ueda P. Seasonal influenza activity during the SARS-CoV-2 outbreak in Japan. JAMA. 2020;323:1969-71. https://doi.org/10.1001/jama.2020.6173

5. Owino CO, Chu JJH. Recent advances on the role of host factors during non-poliovirus enteroviral infections. J Biomed Sci. 2019;26:47. https:/ / doi.org/10.1186/ s12929-019-0540-y

6. Jian SW, Chen CM, Lee CY, Liu DP. Real-time surveillance of infectious diseases: Taiwan's experience. Health Secur. 2017;15:144-53. https:/ / doi.org/10.1089/hs.2016.0107

Address for correspondence: Wei J. Chen, Center for

Neuropsychiatric Research, National Health Research Institutes, No. 35 Keyan Rd, Zhunan, Miaoli 350, Taiwan; email: wjchen@nhri.edu. tw; or Shu-Chen Kuo, National Institute of Infectious Diseases and Vaccinology, National Health Research Institutes, No. 35 Keyan Rd, Zhunan, Miaoli 350, Taiwan; email: sckuo@nhri.edu.tw

\section{Absence of SARS-CoV-2 Transmission from Children in Isolation to Guardians, South Korea}

\author{
Eun Joo Lee, Dong Hyun Kim, Sung Hee Chang, \\ Sun Bok Suh, Jina Lee, Hyunju Lee, Mi Seon Han
}

Author affiliations: Seongnam Citizens Medical Center, Seongnam, South Korea (E.J. Lee); Inha University Hospital, Incheon, South Korea (D.H. Kim); Seoul Medical Center, Seoul, South Korea (S.H. Chang); Busan Medical Center, Busan, South Korea (S.B. Suh); Asan Medical Center, University of Ulsan College of Medicine, Seoul (J. Lee); Seoul National University Bundang Hospital, Seoul National University College of Medicine, Seongnam (H. Lee); Seoul Metropolitan Government-Seoul National University Boramae Medical Center, Seoul (M.S. Han)

DOI: https://doi.org/10.3201/eid2701.203450

We explored transmission of severe acute respiratory syndrome coronavirus 2 among 12 children and their uninfected guardians in hospital isolation rooms in South Korea. We found that, even with close frequent contact, guardians who used appropriate personal protective equipment were not infected by children with diagnosed coronavirus disease.

Oronavirus disease (COVID-19) in children is
known to occur mainly from family clusters (1). However, children can be the only infected members in a household, especially when COVID-19 is contracted from relatives or teachers. Such situations raise concerns about isolation because little information is available on transmission of severe acute respiratory syndrome coronavirus 2 (SARS-CoV-2), which causes COVID-19, during colocation of young children with their uninfected guardians. Although children generally are asymptomatic or have mild symptoms, they could be infective $(1,2)$. We explored whether SARS-CoV-2 was transmitted from children to their uninfected guardians in a hospital isolation setting.

During February 18-June 7, 2020, we analyzed all children $<19$ years of age with COVID-19 and their uninfected guardians who were isolated together in 7 hospitals in South Korea. The infected children were encouraged to wear face masks. The guardians were advised to wear personal protective equipment (PPE), but the degree of PPE varied among hospitals. Adherence to PPE was monitored by the medical staff; compliance was judged as good when PPE was worn most of the time, fair for frequent adherence, 